OPEN ACCESS

Edited by:

Joshua Michael Bauml, University of Pennsylvania,

United States

Reviewed by:

Pinar Ozden Eser,

Dana-Farber Cancer Institute,

United States

Alessandro Russo,

A. O. Papardo, Italy

*Correspondence:

Jie Lin

linjieshi@126.com

Wenhua Liang

liangwh@gird.cn

${ }^{\dagger}$ These authors have contributed equally to this work

Specialty section:

This article was submitted to

Thoracic Oncology,

a section of the journal

Frontiers in Oncology

Received: 10 December 2019 Accepted: 11 September 2020 Published: 02 November 2020

Citation:

Lv L, Liu Z, Liu Y, Zhang W, Jiang L,

Li T, Lu X, Lei $X$, Liang $W$ and Lin J (2020) Distinct EGFR Mutation Pattern in Patients With Non-Small Cell Lung Cancer in Xuanwei Region of China: A Systematic Review and Meta-Analysis.

Front. Oncol. 10:519073.

doi: 10.3389/fonc. 2020.519073

\section{Distinct EGFR Mutation Pattern in Patients With Non-Small Cell Lung Cancer in Xuanwei Region of China: A Systematic Review and Meta-Analysis}

\author{
Li Lv ${ }^{1 \dagger}$, Zhichao Liu ${ }^{2,3,4 \dagger}$, Yang Liu ${ }^{1}$, Wenhui Zhang ${ }^{1}$, Lifeng Jiang ${ }^{1}$, Tingting $\mathrm{Li}^{1}$, \\ Xinyan Lu ${ }^{1}$, Xuefen Lei ${ }^{1}$, Wenhua Liang ${ }^{3,4 *}$ and Jie Lin $^{1 *}$
}

${ }^{1}$ Department of Medical Oncology, The Second Affiliated Hospital of Kunming Medical University, Kunming, China, ${ }^{2}$ School of Medicine, Shanghai Jiao Tong University, Shanghai, China, ${ }^{3}$ Department of Thoracic Surgery and Oncology, The First Affiliated Hospital of Guangzhou Medical University, Guangzhou, China, ${ }^{4}$ Guangzhou Institute of Respiratory Disease and China State Key Laboratory of Respiratory Disease and National Clinical Research Center for Respiratory Disease, Guangzhou, China

Background: In the Xuanwei region of China, lung cancer incidence and mortality are among the highest in China, attributed to severe air pollution generated by combustion of smoky coal. No study has yet comprehensively evaluated the prevalence of epidermal growth factor receptor (EGFR) mutation characteristics in patients with non-small cell lung cancer (NSCLC) in Xuanwei. This meta-analysis was designed to analyze the EGFR mutation pattern in NSCLC patients in Xuanwei region of Yunnan Province in China.

Methods: Electronic databases were comprehensively searched and relevant literatures were retrieved. The odds ratio (OR) for EGFR mutations between Xuanwei region and nonXuanwei region was calculated, and the absolute incidence of EGFR mutations in Xuanwei was pooled by mutation subtype.

Results: Seven studies involving 1,355 patients with NSCLC from Yunnan Province (442 in Xuanwei and 913 in other regions) were included. The EGFR mutation rate ranged between $30.19 \%$ and 55.56\%. Higher uncommon EGFR mutations (OR: 5.69, 95\%Cl: 2.23-14.49, $\mathrm{P}<0.001$ ) and lower common EGFR mutations (OR: 0.18, 95\% Cl: 0.07-0.45, $\mathrm{P}<0.001$ ) were found in Xuanwei region, compared with non-Xuanwei region. Specifically, the uncommon EGFR mutation rate was $59.50 \%$ and common EGFR mutation rate was $40.50 \%$ in Xuanwei. The mutation incidence of exon 18 G719X (OR: 3.21, 95\% Cl: $1.48-$ 6.97, $\mathrm{P}=0.003$ ), exon 20 S768I (OR: 6.44; 95\%Cl: 2.66-15.60; $\mathrm{P}<0.001$ ), and exon 18 G719X + 20 S768I (OR: 6.55; 95\%Cl: 1.92-22.33; P=0.003) in Xuanwei were significantly higher, while the frequency of 19 deletion (OR: $0.28,95 \% \mathrm{Cl}: 0.11-0.77, \mathrm{P}<0.001$ ) and 21 L858R mutation (OR: 0.51, 95\% Cl: 0.31-0.84, $\mathrm{P}=0.007$ ) were lower.

Conclusions: The results highlight the distinct EGFR mutation spectrum of NSCLC patients in Xuanwei region compared with other regions, with higher uncommon 
mutations but lower common mutations. The distinct Xuanwei featured genetic variations provide a unique model to further study carcinogenesis of lung cancer.

Keywords: lung cancer, EGFR mutation, subtype, China, Xuanwei

\section{INTRODUCTION}

Lung cancer has the highest morbidity and is the leading cause of cancer-related death worldwide, with $85 \%$ of patients having nonsmall-cell lung cancer (NSCLC) $(1,2)$. With the advances in molecular oncology, multiple genetic variants have been determined as therapeutic targets for lung cancer, and many onco-targeted drugs had been developed. Epidermal growth factor receptor (EGFR) is a well-accepted carcinogenic variant and driver gene in lung cancer. NSCLC patients with activating EGFR mutations are identified in about $40 \sim 60 \%$ of Asian and $10 \%$ of Western populations (3). When EGFR is activated, it can trigger intracellular signaling cascades that affect cellular proliferation, angiogenesis, and apoptosis through transmembrane receptors (4). Studies showed that EGFR tyrosine kinase inhibitors (TKIs) confer better outcome in patients with the EGFR common mutations (exon 19 deletion, exon 21 L858R point mutation) (5). A genetic divergence of EGFR mutation rates was demonstrated according to ethnicity in previous research $(6,7)$, and the frequency of EGFR was highest among Asians (47\%) and lowest among Oceanians (12\%) (8).

Xuanwei is a small city located in Yunnan Province of China. This region is one of the major coal-producing regions in Yunnan Province and renowned for distinct lung cancer characteristics (9-11): first, lung cancer incidence in Xuanwei region is regionally specific with a high incidence rate and mortality rate. Second, the mortality rate of females is relatively high and almost all of them are never smokers, 20 times higher than other regions of China, and it is among the highest in the world for female lung cancer mortality. Additionally, the onset of lung cancer happens at a relatively younger age in the Xuanwei region, which occurs in younger than the peak age of onset of lung cancer in other parts of China by more than 10 years (12). Environmental factors are known to play a role in lung cancer development, and indoor air pollution from the use of smoky coal for household purposes has been suggested to be the cause of the high incidence of lung cancer in Xuanwei, especially in female patients with non-smoking history (13). Previously, a small-scale study revealed that non-smoking female NSCLC patients in Xuanwei harbored different EGFR mutation patterns when compared with other parts of Asia (14), suggesting that there may exist distinct genetic background in this ethnic group, certain susceptible populations, and unique environments. Therefore, it is of great significance to study the specific EGFR mutation patterns in NSCLC patients from the Xuanwei region of China, which may lead to better understanding the carcinogenesis of EGFR-mutated NSCLC and more effective targeted therapeutic interventions.

The EGFR mutation of NSCLC patients in Xuanwei region has not been fully understood; thus, we conducted this meta- analysis and systematic review to probe further into the EGFR mutation pattern of NSCLC patients in the Xuanwei region compared with the non-Xuanwei population in Yunnan Province to further effectively guide clinical treatment.

\section{METHODS}

\section{Study design}

To obtain a more precise estimate of EGFR mutation pattern in NSCLC patients of Xuanwei region, we pooled the prevalence of EGFR mutation (common and uncommon type) in Xuanwei region and control region (non-Xuanwei) in the rest of Yunnan Province where the level of air pollution and lung cancer incidence and mortality were comparable to most parts of China.

Common EGFR mutations (or classic mutations) are deemed as exon 19 deletion and exon 21 L858R substitution, accounting for approximately $90 \%$ of EGFR mutations in $\operatorname{NSCLC~}(15,16)$. Uncommon EGFR mutations are deemed as mutations other than 19 deletion and 21 L858R, and they account for $10 \%$ to $20 \%$ of all EGFR mutations; the substitution mutations of G719X in exon 18, L861Q in exon 21, S768I in exon 20, exon 20 insertions, and complex mutations are the most frequent among the uncommon mutations (17-19).

\section{Search Strategy}

A comprehensive literature search and systematic review of online databases PubMed, Embase, Web of Science, Medline, Cochrane Library, and Chinese Biomedical Literature Database was performed to identify relevant studies published before 6 November 2019 that examined EGFR mutation frequency in non-small cell lung cancer in southwest China's Yunnan Province. The search key words including "EGFR" AND "mutation" AND ("NSCLC" OR "lung cancer") AND ("Yunnan" OR "Xuanwei") were used. No search limitations were set. The relevant abstracts and presentations from conferences were also manually searched. Then, we examined the publication of the list of references and searched for additional research.

\section{Inclusion and Exclusion Criteria}

Eligible studies should meet the following criteria: (i) Publications describing the mutation subtype in EGFR-mutated NSCLC of Yunnan in southwestern China were retained. (ii) Mutation detection in paraffin-embedded tumor tissues or cytological specimens or blood samples can be included.

Studies were excluded if (i) data were insufficient to calculate the pooled incidence for this meta-analysis or (ii) they were review 
articles, case reports, editorials, expert opinions, non-comparative studies, unrelated to research topics, or duplicate reports.

\section{Data Extraction and Quality Assessment}

This meta-analysis was conducted according to the Cochrane and Preferred Reporting Items for Systematic Reviews and MetaAnalyses guidelines (20). Two researchers (Li Lv and Zhichao Liu) independently performed all of the screening of studies and data extraction. The third researcher (Yang Liu) resolved the disagreements. For eligible research, we extract all available information: the first name of the author, year of publication, region, research type, number of patients, gender, age, smoking status, EGFR mutations, detection methods, tumor histology, disease stage, and metastases type. The quality of the included studies was assessed using the Agency for Healthcare Research and Quality Tool. Any disagreements were resolved through discussion and consensus.

\section{Statistical Analysis}

Pooled odds ratio (OR) of common and uncommon EGFR mutation rate between Xuanwei region and non-Xuanwei region in Yunnan province of China was calculated, and the pooled frequency of EGFR mutations in Xuanwei was also calculated. Subgroup pooled OR and incidence were generated according to EGFR mutation subtypes. Cochran's Q test and $I^{2}$ were used to estimate the heterogeneity effect among the studies. $I^{2}$ statistics more than $50 \%$ was suggestive of statistical heterogeneity between studies, and random effects model was used if heterogeneity existed. All tests were two-sided and a $\mathrm{P}$ value less than 0.05 was considered statistically significant. All statistical analyses were conducted with STATA 12.0 software (Stata Corporation, College Station, TX, USA) and R 3.4.1 software ( $\mathrm{R}$ foundation for Statistical Computing, Vienna, Austria).

\section{RESULTS}

\section{The Selection and Characteristics of Study}

A total of 1,473 publications were retrieved through the initial literature search, of which 658 papers were excluded due to duplication. With title and abstract review, 10 potentially relevant articles were identified for detailed review (13, 14, 21 28). These articles were further assessed for eligibility by reviewing the full texts, and 3 articles (23-25) were removed due to insufficient data. Finally, a total of 7 articles (13, 14, 21, 22, 26-28) were identified as eligible to be included in the metaanalysis (Figure 1).

Table 1 summarized the characteristics of all included studies. Seven studies involving 1,355 NSCLC patients from different regions of Yunnan Province in southwestern China (442 from Xuanwei region, 913 from other region in the rest of Yunnan Province) were included. The majority of patients were adenocarcinoma and non-smoker. Four studies provided a comparison of EGFR mutations between Xuanwei and nonXuanwei regions, while the other 3 studies $(14,26,27)$ only

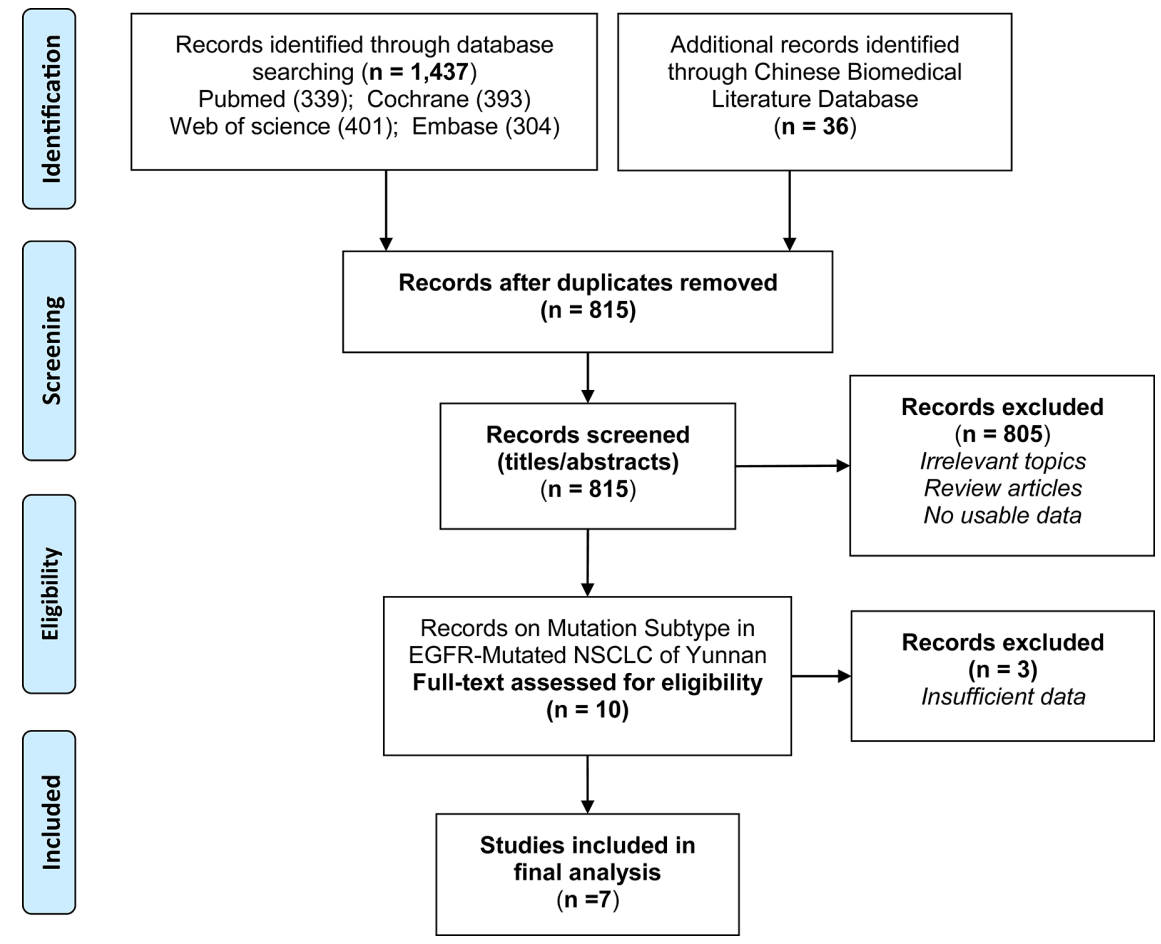

FIGURE 1 | Flow chart detailing the search strategy and identification of studies. 


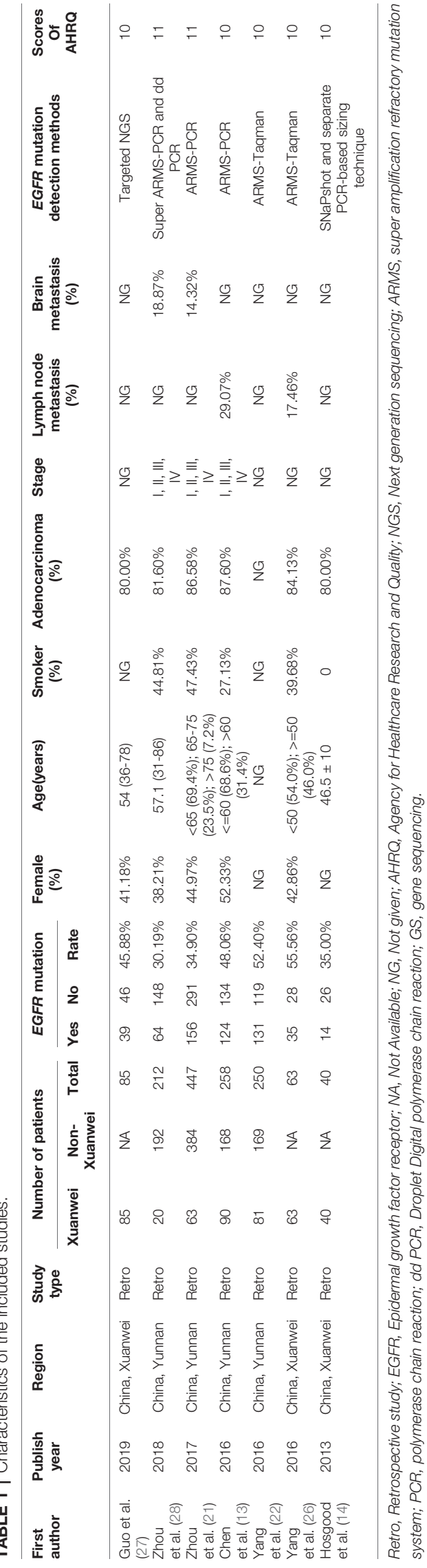

evaluated EGFR mutations in Xuanwei region. In all included studies $(13,14,21,22,26-28)$, the EGFR mutation status was mainly (5 out of 7) detected by Amplification Refractory Mutation System (ARMS), while next generation sequencing (NGS) or SNaPshot were used in the other 2 studies. Overall, the EGFR mutation positive rate was $30.19 \%$ to $55.56 \%$ across studies. All studies gained 10 to 11 scores in study quality assessment on a scale of 0 to 11 with the Agency for Healthcare Research and Quality Tool.

\section{Frequency and Odds Ratio of EGFR Mutation in Xuanwei and Non-Xuanwei Regions}

Four studies $(13,21,22,28)$ that simultaneously reported the EGFR mutation rate in Xuanwei and non-Xuanwei region were included in the comparative analysis. The difference in EGFR mutation rates is summarized according to regions (Table 2). One hundred and twenty-nine patients from Xuanwei region harbored EGFR mutations, with $64.34 \%(83 / 129)$ uncommon mutation and $35.66 \%(46 / 129)$ common mutation. In non-Xuanwei region, the incidence of uncommon and common EGFR mutation was $25.43 \%$ (88/346) and $74.57 \%(258 / 346)$ respectively. The frequency of uncommon mutations was higher in Xuanwei region than that in non-Xuanwei region (4 studies $(13,21,22,28)$ OR: $5.69,95 \% \mathrm{CI}$ : 2.23-14.49, $\mathrm{P}<0.001$ ) (Figure 2B). By contrast, patients in Xuanwei regions were less likely to have common EGFR mutations (19 deletion or $21 \mathrm{~L} 858 \mathrm{R}$ ) compared with non-Xuanwei region (OR: 0.18, 95\%CI: 0.07-0.45, $\mathrm{P}<0.001$ ) (Figure 2A).

\section{Overall Incidence of Common and Uncommon EGFR Mutation in Xuanwei Region}

To further illustrate the distribution of common and uncommon EGFR mutations in Xuanwei region, 7 studies (13, 14, 21, 22, 2628) were included to calculate the pooled incidence (Table 3). A total of 217 patients had EGFR mutation in Xuanwei region. The pooled incidence of uncommon EGFR mutation was 59.5\% (95\% CI: 53.2\%-65.9\%), while the pooled incidence of common EGFR mutation was $40.5 \%$ (95\%CI: $34.1 \%-46.8 \%)$.

\section{Subgroup Analysis of Different EGFR Mutation Subtypes in Xuanwei and Non- Xuanwei Regions}

Patients from Xuanwei and non-Xuanwei regions with specific EGFR mutation subtypes were further analyzed (Table 4). Results showed that mutation of 18 G719X, 20 S768I, and 18 G719X + 20 S768I were more likely to appear in Xuanwei patients compared with non-Xuanwei patients (OR: 3.21, 95\% CI: 1.48-6.97, $\mathrm{P}=0.003$; OR: 6.44, 95\%CI: $2.66-15.60, \mathrm{P}<0.001$, OR: $6.55,95 \%$ CI: $1.92-22.33, \mathrm{P}<0.05$, respectively). In contrast, NSCLC patients of Xuanwei regions harbored lower frequency of 19 deletion (OR: 0.28, 95\%CI: 0.11-0.77, $\mathrm{P}<0.001)$ and $21 \mathrm{~L} 858 \mathrm{R}$ mutation (OR: 0.51, 95\%CI: 0.31-0.84, $\mathrm{P}=0.007$ ). 
TABLE 2 | Comparison of the incidence of EGFR mutation of NSCLC patients between Xuanwei and non-Xuanwei regions.

\begin{tabular}{|c|c|c|c|c|c|c|c|c|c|c|c|c|c|}
\hline & & & \multicolumn{4}{|c|}{ Xuanwei } & \multicolumn{4}{|c|}{ Non-Xuanwei } & \multicolumn{3}{|c|}{ META } \\
\hline & & & Yes & No & Total & Rate & Yes & No & Total & Rate & OR & $95 \% \mathrm{Cl}$ & $P$-value \\
\hline \multirow[t]{6}{*}{ Common mutation } & Zhou et al. (28) & 2018 & 3 & 5 & 8 & $37.50 \%$ & 28 & 28 & 56 & $50.00 \%$ & 0.600 & $(0.131-2.755)$ & \\
\hline & Zhou et al. (21) & 2017 & 8 & 19 & 27 & $29.63 \%$ & 101 & 28 & 129 & $78.29 \%$ & 0.117 & $(0.046-0.295)$ & \\
\hline & Chen et al. (13) & 2016 & 14 & 37 & 51 & $27.45 \%$ & 63 & 10 & 73 & $86.30 \%$ & 0.060 & (0.024-0.149) & \\
\hline & Yang et al. (22) & 2016 & 21 & 22 & 43 & $48.84 \%$ & 66 & 22 & 88 & $75.00 \%$ & 0.318 & $(0.148-0.686)$ & \\
\hline & Overall & & 46 & 83 & 129 & & 258 & 88 & 346 & & 0.176 & $(0.069-0.448)$ & $<0.001$ \\
\hline & & & & & & & & & & & \multicolumn{3}{|c|}{ (Heterogeneity: $\left.l^{2}=72.4 \%, p=0.0012\right)$} \\
\hline \multirow[t]{6}{*}{ Uncommon mutation } & Zhou et al. (28) & 2018 & 5 & 3 & 8 & $62.50 \%$ & 28 & 28 & 56 & $50.00 \%$ & 1.667 & $(0.363-7.652)$ & \\
\hline & Zhou et al. (21) & 2017 & 19 & 8 & 27 & $70.37 \%$ & 28 & 101 & 129 & $21.71 \%$ & 8.567 & (3.393-21.628) & \\
\hline & Chen et al. (13) & 2016 & 37 & 14 & 51 & $72.55 \%$ & 10 & 63 & 73 & $13.70 \%$ & 16.650 & $(6.720-41.256)$ & \\
\hline & Yang et al. (22) & 2016 & 22 & 21 & 43 & $51.16 \%$ & 22 & 66 & 88 & $25.00 \%$ & 3.143 & $(1.458-6.777)$ & \\
\hline & Overall & & 83 & 46 & 129 & & 88 & 258 & 346 & & 5.685 & $(2.231-14.486)$ & $<0.001$ \\
\hline & & & & & & & & & & & \multicolumn{3}{|c|}{ (Heterogeneity: $\left.P^{2}=72.4 \%, p=0.0012\right)$} \\
\hline
\end{tabular}

OR, odds ratio; $\mathrm{Cl}$, confidence interval.

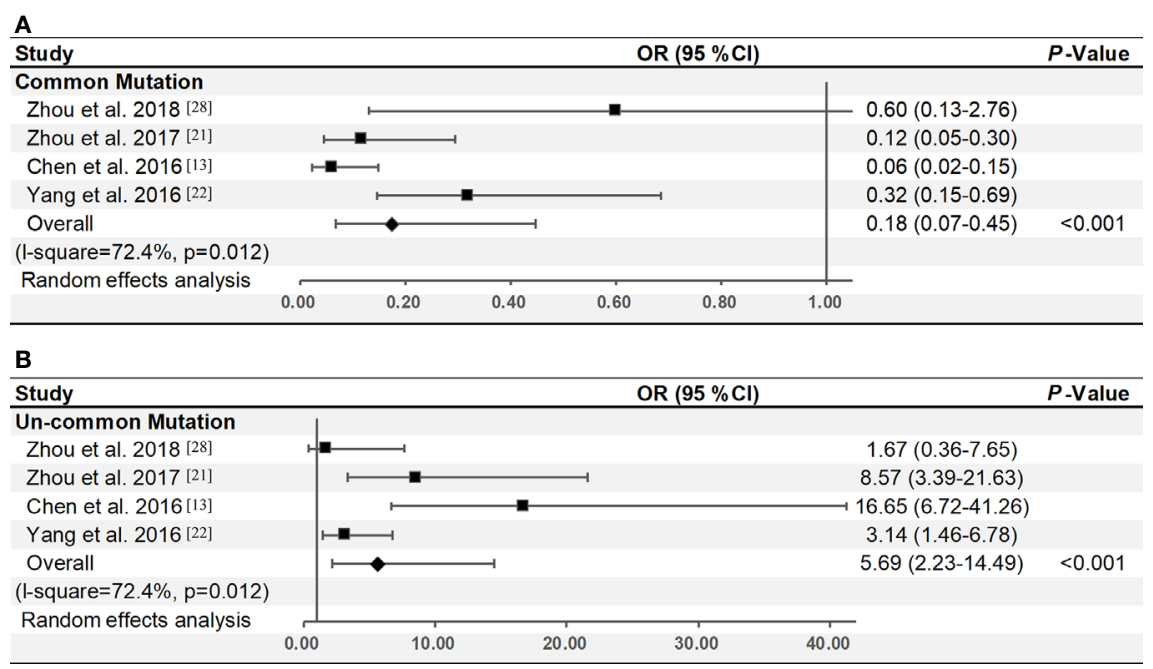

FIGURE 2 | Odds of common (A) or uncommon (B) EGFR mutation of Xuanwei compared to non-Xuanwei regions (four studies). The center of each square is the odds ratio (OR) for individual trials and corresponding horizontal line is the $95 \% \mathrm{Cl}$. The broken line and center of the blue diamond is overall pooled OR and the horizontal tip of the diamond is the $95 \%$ confidence interval $(\mathrm{Cl})$.

\section{DISCUSSION}

Due to the low incidence of NSCLC with the so-called uncommon EGFR mutations in the general population, information on their significance of carcinogenesis and treatment is still incomplete and deserves further investigation. We conducted a systematic review and meta-analysis of current researches $(13,14,21,22,26-28)$ to evaluate the mutation pattern of EGFR-mutated NSCLC of Yunnan province in southwestern China. The pooled analysis confirmed a distinct EGFR mutation spectrum in Xuanwei region. The NSCLC patients in Xuanwei region are present with significantly higher incidence of uncommon EGFR mutations, especially 18 G719X mutation, 20 S768I mutation, and their combined mutation, but lower incidence of the two common mutations (19 deletion and 21 L858R substitution), providing a unique model for uncommon-EGFR-mutation-related lung cancer.
EGFR mutations in NSCLC is one of the most common genetic variations, especially in East Asians, females, and nonsmokers (29). Studies suggested that NSCLC patients with EGFR mutation were significantly related to adenocarcinoma and light smoking, rather than gender (30). It was suggested that the dominant mutation rate of EGFR in women is a reflection of a higher frequency of adenocarcinoma (31). The results of our analysis revealed that the overall EGFR mutation rate of NSCLC patients varied from $30.2 \%$ to $55.6 \%$ in Yunnan province (Xuanwei region: 40.0\%-56.7\%; non-Xuanwei region: 29.2\%$52.1 \%$ ), which was in the range of other reports in East Asian areas $(31 \%-56 \%)(8,31-34)$ and similar to other studies performed in other regions of China (ranging from $33.64 \%$ to 53.69\%)(Table S1). Most patients covered by our survey were carriers of adenocarcinomas (ranging from $80.0 \%$ to $87.6 \%$ ) and female (ranging from $38.2 \%$ to $52.3 \%$ ), which was similar to 
TABLE 3 | Incidence of common and uncommon EGFR mutation in Xuanwei region.

\begin{tabular}{|c|c|c|c|c|c|c|c|c|}
\hline & & & \multicolumn{4}{|c|}{ Xuanwei } & \multicolumn{2}{|c|}{ META } \\
\hline & & & Yes & No & Total & Rate & Rate & $95 \% \mathrm{Cl}$ \\
\hline \multirow[t]{9}{*}{ Common mutation } & Guo et al. (27) & 2019 & 22 & 17 & 39 & $56.41 \%$ & 0.564 & $(0.408-0.720)$ \\
\hline & Zhou et al. (28) & 2018 & 3 & 5 & 8 & $37.50 \%$ & 0.375 & $(0.040-0.710)$ \\
\hline & Zhou et al. (21) & 2017 & 8 & 19 & 27 & $29.63 \%$ & 0.296 & $(0.124-0.469)$ \\
\hline & Chen et al. (13) & 2016 & 14 & 37 & 51 & $27.45 \%$ & 0.275 & $(0.152-0.397)$ \\
\hline & Yang et al. (22) & 2016 & 21 & 22 & 43 & $48.84 \%$ & 0.488 & (0.339-0.638) \\
\hline & Yang et al. (26) & 2016 & 16 & 19 & 35 & $45.71 \%$ & 0.457 & $(0.292-0.622)$ \\
\hline & Hosgood et al. (14) & 2013 & 6 & 8 & 14 & $42.86 \%$ & 0.429 & $(0.169-0.688)$ \\
\hline & Overall & & 90 & 127 & 217 & & 0.405 & $(0.341-0.468)$ \\
\hline & & & & & & & & $\left(\right.$ Heterogeneity: $\left.l^{2}=48.0 \%, p=0.073\right)$ \\
\hline \multirow[t]{9}{*}{ Uncommon mutation } & Guo et al. (27) & 2019 & 17 & 22 & 39 & $43.59 \%$ & 0.436 & (0.280-0.592) \\
\hline & Zhou et al. (28) & 2018 & 5 & 3 & 8 & $62.50 \%$ & 0.625 & $(0.290-0.960)$ \\
\hline & Zhou et al. (21) & 2017 & 19 & 8 & 27 & $70.37 \%$ & 0.704 & $(0.531-0.876)$ \\
\hline & Chen et al. (13) & 2016 & 37 & 14 & 51 & $72.55 \%$ & 0.725 & $(0.603-0.848)$ \\
\hline & Yang et al. (22) & 2016 & 22 & 21 & 43 & $51.16 \%$ & 0.512 & $(0.362-0.661)$ \\
\hline & Yang et al. (26) & 2016 & 19 & 16 & 35 & $54.29 \%$ & 0.543 & $(0.378-0.708)$ \\
\hline & Hosgood et al. (14) & 2013 & 8 & 6 & 14 & $57.14 \%$ & 0.571 & $(0.312-0.831)$ \\
\hline & Overall & & 127 & 90 & 217 & & 0.595 & $(0.532-0.659)$ \\
\hline & & & & & & & & (Heterogeneity: $\left.l^{2}=48.0 \%, p=0.073\right)$ \\
\hline
\end{tabular}

other studies performed in East Asian countries (8, 33, 34). Notably, despite similar incidence of overall EGFR mutations, the NSCLC patients in Xuanwei region present a distinctive characteristic with higher uncommon EGFR mutations and lower common EGFR mutations (Table 2, Table S2).

Generally, exon 19 deletions and 21 L858R substitutions, accounting for approximately $90 \%$ of EGFR mutations in NSCLC, are termed common or classic mutations and lead to high sensitivity to EGFR TKIs (35). Other EGFR mutations are termed uncommon mutations, accounting for $10 \%-20 \%$ of all EGFR mutations, and patients with uncommon EGFR mutation are a heterogeneous group exhibiting different responses to EGFR TKIs $(36,37)$. There are significant regional differences in EGFR gene mutation in China. It has been reported that exon 21 of EGFR gene mutation is dominant in Taiwan (38), while exon 19 is dominant in Yunnan (21) and exon 20 is dominant in Guangdong (36). Our results show that EGFR gene mutation mainly occurred in exon 21 L858R mutation and in exon 19 deletion NSCLC patients in most regions of Yunnan, which was consistent with other reports. Another study showed that the uncommon EGFR mutations were present in $11.9 \%$ of all patients with documented EGFR mutation (34\%) in a Chinese NSCLC cohort (36). However, according to our study, the detection rate of uncommon EGFR mutations varied from $43.6 \%$ to $72.6 \%$ in NSCLC patients in Xuanwei, which was significantly higher than that of the general Chinese population and other regions of the world (ranging from $3.4 \%$ to $15.5 \%$ ) (Table 2, Table S2). This result further emphasizes the uniqueness of the Xuanwei population in lung cancer tumorigenesis.

The majority of clinical trials evaluating the efficacy of EGFR TKIs have included only patients with common EGFR mutations due to the low incidence of uncommon EGFR mutations in the general population; thus, the efficacy of EGFR TKIs in patients with uncommon EGFR mutations remains elusive. A post-hoc study showed that NSCLC patients with uncommon EGFR mutation (L861Q or G719X) was significantly associated with shorter overall survival (11.9 vs. 29.3 months) compared with patients harboring common EGFR mutation (L858R or 19 deletion) in the EGFR TKI treatment group (39). The patients with uncommon EGFR mutation (41\% vs. $62 \%-83 \%)$ had a significantly lower overall response rate (ORR) of EGFR TKIs and shorter progression-free survival (PFS) (2.2 vs. 11.4 months) than that in those with common EGFR mutation (39). Additionally, in other studies, a poor ORR and shorter PFS were also found in uncommon EGFR mutations compared with the classic EGFR mutations. However, a similarly poor response to EGFR TKIs was observed in other uncommon mutation rather than G719X and L861Q $(31,40)$. It has also been documented that patients with NSCLC EGFR mutation in Xuanwei have poor prognosis after treatment with EGFR TKIs (21), possibly owing to the high incidence of uncommon EGFR mutations in this area.

Recent studies have suggested that the lung cancer in Xuanwei region showed distinct mutational signatures and signaling pathways, and two single nucleotide variants located in exon 20 and exon 18 of the EGFR gene were known to be related to lung adenocarcinoma in Xuanwei (41). In line with this, our results also suggested that patients with exon 20 S768I and exon 18 G719X mutations presented higher mutation rates than those from other regions. The specific genotypes of complex EGFR mutations (double or multiple concomitant EGFR mutations) are diverse, which can be common single mutation combined with common single mutation, common single mutation combined with rare single mutation, rare 


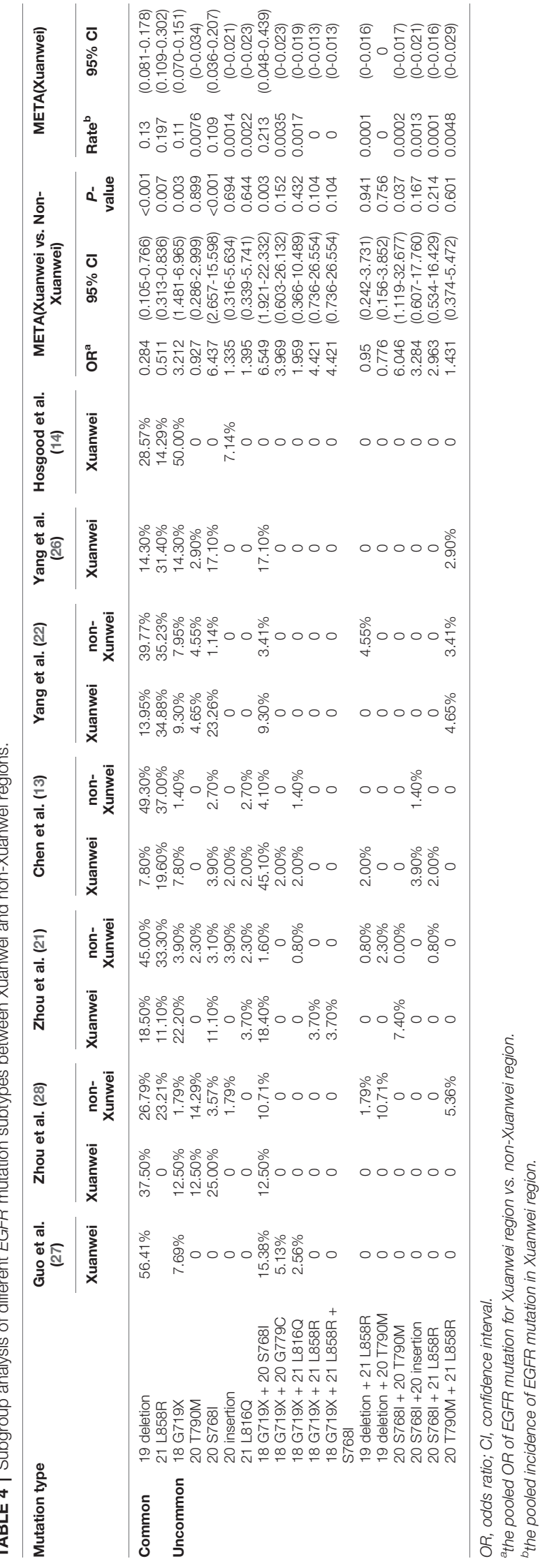

single mutation combined with rare single mutation, or compound mutation combined with known resistance genes. In this study, in patients with complex mutations, there was only one case with the common mutation combination (19deletion + L858R), while the others were the rare mutation combination, mainly including G719X, S768I, T790M, 20 insertions, and L861Q. Specifically, the 20 S768I mutation, 18 G719X mutation, co-mutation in 20 S768I + 18 G719X, and co-mutation in 20 S768I + 20 T790M were significantly more likely to present in Xuanwei patients compared with non-Xuanwei patients (all $P<0.05$ ). These mutations were thought to promote the development and progression of lung cancer (42). For other uncommon EGFR mutation types, there was no statistically significant difference between Xuanwei and nonXuanwei regions. However, it should be noted that some comutations with uncommon EGFR mutation did present an unnegligible high OR value in Xuanwei region, such as $18 \mathrm{G} 719 \mathrm{X}+21$ L858R (OR: 4.42), 18 G719X + 20 G779C (OR: 3.97), and 20 S768I +20 insertion (OR: 3.28). In other ways, the 20 S768I + 20 T790M was significantly more frequently present in Xuanwei region (OR, 6.05) whereas 19 deletion +20 T790M tends to be present less in Xuanwei region (OR, 0.77), which may reflect an acquired resistance after EGFR-TKI therapy in these NSCLC patients with prevalent uncommon EGFR mutation in Xuanwei region. Overall, these results revealed a relatively high mutation frequency of socalled uncommon mutations (e.g., 18 G719X, 20 S768I, or 18 G719X/20 S768I in conjunction with other mutation) in the unique Xuanwei population, strikingly divergent from those in other populations from Asia. Given that our subjects (Xuanwei population) live in an area where coal is typically burned indoors, our analysis implies that the tumorigenesis and progression of lung cancer in Xuanwei region is different from that in other geographic areas, which may due to its distinctive etiology and the different environmental exposures.

The main difference between environmental exposure may be related to indoor solid fuel use related to indoor air pollution. In Xuanwei, indoor air pollution from bituminous coal burning in unvented fire pits was suggested to be the main cause of high lung cancer mortality (11). Polycyclic aromatic hydrocarbons (PAHs)DNA adducts have been observed in the bronchoalveolar lavage fluid of coal-burning residents in Xuanwei region (11). Previous study showed that the mutations in EGFR exons 21 and 18 were associated with emissions from coal combustion (14). In addition, PAHs have been found to increase intracellular calcium in human cells (43), which may result in EGFR-dependent cell proliferation (4), suggesting that PAHs may lead to a unique mutation pattern. Liu et al. (44) evaluated the relationship among indoor air pollution, tobacco use, and lung cancer risk, showing that the risk association between smoking and lung cancer increased with the decrease of bituminous coal consumption. In other words, the relationship between smoking and lung cancer is relatively weak when there is a strong correlation between lung cancer and bituminous coal. Xuanwei is located in one of China's largest tobacco producing provinces (45). In Xuanwei, the smoking rates of males in high, medium, and low incidence areas were $75 \%, 78 \%$, and $63 \%$ respectively, and the exposure rates of second-hand smoke were $85 \%, 88 \%$, and $58 \%$, which were much higher than the national 
average level of male smoking rate $(52.1 \%)$ and second-hand smoke exposure level (72.4\%) (46). The discrepancy of mutation subtypes may provide clues for the mechanism of the occurrence of EGFR mutation. And this comparative analysis provides reference data, allowing a better understanding of a possible mechanism of EGFR mutation in Xuanwei, paving the way toward better exploration of uncommon EGFR mutation in lung cancer pathogenesis.

There are several limitations to the study. First, all included publications were retrospective studies with inherent biases. Second, the sample size of the study was not big enough. Third, only one study used NGS method to detect EGFR mutation; most of the included studies applied ARMS method to detect EGFR mutation, which is limited to the detection of known mutations and therefore might miss some rare EGFR mutations. Nevertheless, it should be noted that the detection methods in this analysis were already sufficient for the detection of classic (19 deletions and 21 L858R) and those known nonclassic EGFR mutations in exons 18 to 21. A high concordance was found between ARMS and NGS, but more detailed information (unknown genetic mutations and deep sequencing) could be revealed by NGS (30, 47, 48). Therefore, a larger amount of sample and adoption of NGS is needed to validate the results of this study.

In conclusion, our analysis suggested the prevalence of EGFR mutation in Xuanwei region that is differentiating it from the general population. The frequency of S768I, G719X, and G719X +S768I were higher, but the 19 deletions and L858R mutations were lower in Xuanwei region. The difference of EGFR mutation between patients in Xuanwei region and in other areas may indicate the difference of lung cancer pathogenesis, dietary habits, coal-burning factors, or genetic backgrounds, which are worthy of more detailed studies.

\section{REFERENCES}

1. Bray F, Ferlay J, Soerjomataram I, Siegel RL, Torre LA, Jemal A. Global cancer statistics 2018: Globocan estimates of incidence and mortality worldwide for 36 cancers in 185 countries. CA: Cancer J Clin (2018) 68:394-424. doi: 10.3322/ caac. 21492

2. Chen W, Zheng R, Zhang S, Zeng H, Xia C, Zuo T, et al. Cancer incidence and mortality in china, 2013. Cancer Lett (2017) 401:63-71. doi: 10.1016/ j.canlet.2017.04.024

3. Pao W, Girard N. New driver mutations in non-small-cell lung cancer. Lancet Oncol (2011) 12:175-80. doi: 10.1016/S1470-2045(10)70087-5

4. Scaltriti M, Baselga J. The epidermal growth factor receptor pathway: A model for targeted therapy. Clin Cancer Res (2006) 12:5268-72. doi: 10.1158/10780432.CCR-05-1554

5. Baek JH, Sun JM, Min YJ, Cho EK, Cho BC, Kim JH, et al. Efficacy of EGFR tyrosine kinase inhibitors in patients with EGFR-mutated non-small cell lung cancer except both exon 19 deletion and exon 21 1858r: A retrospective analysis in korea. Lung Cancer (Amsterdam Netherlands) (2015) 87:148-54. doi: 10.1016/j.lungcan.2014.11.013

6. Zhou W, Christiani DC. East meets west: Ethnic differences in epidemiology and clinical behaviors of lung cancer between east asians and caucasians. Chin J Cancer (2011) 30:287-92. doi: 10.5732/cjc.011.10106

7. Midha A, Dearden S, McCormack R. EGFR mutation incidence in non-smallcell lung cancer of adenocarcinoma histology: A systematic review and global map by ethnicity (mutmapii). Am J Cancer Res (2015) 5:2892-911.

8. Shi Y, Au JS, Thongprasert S, Srinivasan S, Tsai CM, Khoa MT, et al. A prospective, molecular epidemiology study of EGFR mutations in asian

\section{DATA AVAILABILITY STATEMENT}

The raw data supporting the conclusions of this article will be made available by the authors, without undue reservation, to any qualified researcher.

\section{AUTHOR CONTRIBUTIONS}

JL and WL designed and financed the study. LL performed the literature search and review, data extraction, and drafted the manuscript. ZL performed all of the screening of studies and data extraction. Any disagreements were resolved by YL. WZ, LJ, and TL were responsible for the analysis of pooled data. All authors contributed to the article and approved the submitted version.

\section{FUNDING}

This study was supported by the Research of Yunnan Provence Science and Technology Planning Project (Project No.2017FE468 (-201), Project No.202001AT070027); the National Natural Science Foundation of China (Project No. 81960423) and the Second Affiliated Hospital of Kunming Medical University Program (grant no. 2019YK001).

\section{SUPPLEMENTARY MATERIAL}

The Supplementary Material for this article can be found online at: https://www.frontiersin.org/articles/10.3389/fonc.2020. 519073/full\#supplementary-material

patients with advanced non-small-cell lung cancer of adenocarcinoma histology (pioneer). J Thoracic Oncol (2014) 9:154-62. doi: 10.1097/ JTO.0000000000000033

9. Ren H, Cao W, Chen G, Yang J, Liu L, Wan X, et al. Lung cancer mortality and topography: A xuanwei case study. Int J Environ Res Public Health (2016) 13:473. doi: 10.3390/ijerph13050473

10. Wong JYY, Downward GS, Hu W, Portengen L, Seow WJ, Silverman DT, et al. Lung cancer risk by geologic coal deposits: A case-control study of female never-smokers from xuanwei and fuyuan, china. Int J Cancer (2019) 144:2918-27. doi: 10.1002/ijc.32034

11. Hosgood HD 3rd, Mongodin EF, Wan Y, Hua X, Rothman N, Hu W, et al. The respiratory tract microbiome and its relationship to lung cancer and environmental exposures found in rural china. Environ Mol Mutagen (2019) 60:617-23. doi: 10.1002/em.22291

12. Li J, Zhang Y, Li Y, Yin G, Li Y, Ning B, et al. Descriptive study on the epidemiology of lung cancer in coal-producing area in eastern Yunnan, China. Zhongguo Fei Ai Za Zhi (2011) 14(2):107-19. doi: 10.3779/j.issn.1009-3419

13. Chen Y, Ye L, Stanford RR, Zhang D, Zhang X, Wei W. Distinct epithelial growth factor receptor mutation profile in non-small-cell lung cancer patients from the xuanwei area of china. Mol Clin Oncol (2016) 4:749-55. doi: 10.3892/mco.2016.805

14. Hosgood HD 3rd, Pao W, Rothman N, Hu W, Pan YH, Kuchinsky K, et al. Driver mutations among never smoking female lung cancer tissues in China identify unique EGFR and KRAS mutation pattern associated with household coal burning. Respir Med (2013) 107(11):1755-62. doi: 10.1016/j.rmed

15. Rosell R, Moran T, Queralt C, Porta R, Cardenal F, Camps C, et al. Screening for epidermal growth factor receptor mutations in lung cancer. $N$ Engl J Med (2009) 361(10):958-67. doi: 10.1056/NEJMoa0904554 
16. Lee CK, Brown C, Gralla RJ, Hirsh V, Thongprasert S, Tsai CM, et al. Impact of EGFR inhibitor in non-small cell lung cancer on progression-free and overall survival: a meta-analysis. J Natl Cancer Inst (2013) 105(9):595-605. doi: $10.1093 /$ jnci/djt072

17. Rosell R, Carcereny E, Gervais R, Vergnenegre A, Massuti B, Felip E, et al. Erlotinib versus standard chemotherapy as first-line treatment for European patients with advanced EGFR mutation-positive non-small-cell lung cancer (EURTAC): a multicentre, open-label, randomised phase 3 trial. Lancet Oncol (2012) 13(3):239-46. doi: 10.1016/S1470-2045(11)70393-X

18. Mok TS, Wu YL, Thongprasert S, Yang CH, Chu DT, Saijo N, et al. Gefitinib or carboplatin-paclitaxel in pulmonary adenocarcinoma. N Engl J Med (2009) 361(10):947-57. doi: 10.1056/NEJMoa0810699

19. Evans M, O'Sullivan B, Smith M, Hughes F, Mullis T, Trim N, et al. LargeScale EGFR Mutation Testing in Clinical Practice: Analysis of a Series of 18,920 Non-Small Cell Lung Cancer Cases. Pathol Oncol Res (2019) 25 (4):1401-9. doi: 10.1007/s12253-018-0460-2

20. Liberati A, Altman DG, Tetzlaff J, Mulrow C, Gøtzsche PC, Ioannidis JP, et al. The prisma statement for reporting systematic reviews and meta-analyses of studies that evaluate healthcare interventions: Explanation and elaboration. BMJ (2009) 339:b2700. doi: 10.1136/bmj.b2700

21. Zhou Y, Yang Y, Yang C, Chen Y, Yang C, Du Y, et al. Epidermal growth factor receptor (EGFR) mutations in non-small cell lung cancer (NSCLC) of yunnan in southwestern china. Oncotarget (2017) 8:15023-33. doi: 10.18632/ oncotarget. 14706

22. Yang CS, Xu WM, Feng Q, Wang YY, Pan XY, Wang L, et al. EGFR gene mutation of non-small cell lung cancer from different regions of Yunnan Province. Xinan Guo Fang Yi Yao (2016) 26:4-7. doi: 10.3969/j.issn.1004-0188.2016.01.002

23. Su GM, Yang Z, Bian L, Pan GQ. Study on mutation of EGFR gene in non-small cell lung cancers in Kunming of Yunnan. Fen Zi Zhen Duan Yu Zhi Liao Za Zhi (2016) 8:321-4. doi: 10.3969/j.issn.1674-6929.2016.05.006

24. Hosgood HD 3rd, Pao W, Rothman N, Hu W, Pan YH, Kuchinsky K, et al. Somatic driver mutations among never smoking female lung cancer cases in china identify unique mutation pattern that may be associated with household coal burning. Cancer Res (2012) 72:5479-9. doi: 10.1016/j.rmed.2013.08.018

25. Shen M, Vermeulen R, Rajaraman P, Menashe I, He X, Chapman RS, et al. Polymorphisms in innate immunity genes and lung cancer risk in xuanwei, china. Environ Mol Mutagen (2009) 50:285-90. doi: 10.1002/em.20452

26. Yang CS, Pan XY, Feng Q, Wang YY, Xu WM, Jiang T, et al. mutation status of epidermal growth factor receptor and kras gene in non-small cell lung cancers at xuanwei regions of Yunnan Province. Zhonghua Bing Li Xue Za Zhi (2016) 45:226-30. doi: 10.3760/cma.j.issn.0529-5807.2016.04.003

27. Guo G, Shou T, Li G, Peng H, Zhao J, Guo H, et al. Genomic profiling of nonsmall cell lung cancer in xuanwei, Yunnan Province. Cancer Res (2019) 79:3411. doi: 10.1158/1538-7445.SABCS18-3411

28. Zhou Y, Ma Y, Shi H, Du Y, Huang Y. Epidermal growth factor receptor t790m mutations in non-small cell lung cancer (nsclc) of yunnan in southwestern china. Sci Rep (2018) 8:15426. doi: 10.1038/s41598-018-33816-x

29. Kosaka T, Yatabe Y, Endoh H, Kuwano H, Takahashi T, Mitsudomi T. Mutations of the epidermal growth factor receptor gene in lung cancer: Biological and clinical implications. Cancer Res (2004) 64:8919-23. doi: 10.1158/0008-5472.CAN-04-2818

30. Vendrell JA, Mau-Them FT, Béganton B, Godreuil S, Coopman P, Solassol J. Circulating Cell Free Tumor DNA Detection as a Routine Tool forLung Cancer Patient Management. Int J Mol Sci (2017) 18(2):264. doi: 10.3390/ ijms18020264

31. Tanaka T, Matsuoka M, Sutani A, Gemma A, Maemondo M, Inoue A, et al. Frequency of and variables associated with the EGFR mutation and its subtypes. Int J Cancer (2010) 126:651-5. doi: 10.1002/ijc.24746

32. Lee SH, Kim WS, Choi YD, Seo JW, Han JH, Kim MJ, et al. Cardiopulmonary Pathology Study Group of Korean Society of Pathologists. Analysis of Mutations in Epidermal Growth Factor Receptor Gene in Korean Patients with Non-small Cell Lung Cancer: Summary of a Nationwide Survey. J Pathol Transl Med (2015) 49(6):481-8. doi: 10.4132/jptm

33. Liam CK, Wahid MI, Rajadurai P, Cheah YK, Ng TS. Epidermal growth factor receptor mutations in lung adenocarcinoma in Malaysian patients. $J$ Thorac Oncol (2013) 8(6):766-72. doi: 10.1097/JTO.0b013e31828b5228
34. Shi Y, Li J, Zhang S, Wang M, Yang S, Li N, et al. Molecular Epidemiology of EGFR Mutations in Asian Patients with Advanced Non-Small-Cell Lung Cancer of Adenocarcinoma Histology - Mainland China Subset Analysis of the PIONEER study. PloS One (2015) 10(11):e0143515. doi: 10.1371/journal.pone.0143515

35. Castellanos E, Feld E, Horn L. Driven by mutations: The predictive value of mutation subtype in EGFR-mutated non-small cell lung cancer. $J$ Thoracic Oncol Off Publ Int Assoc Study Lung Cancer (2017) 12:612-23. doi: 10.1016/ j.jtho.2016.12.014

36. Tu HY, Ke EE, Yang JJ, Sun YL, Yan HH, Zheng MY, et al. A comprehensive review of uncommon EGFR mutations in patients with non-small cell lung cancer. Lung Cancer (2017) 114:96-102. doi: 10.1016/j.lungcan.2017.11.005

37. Wu TH, Hsiue EH, Lee JH, Lin CC, Yang JC. New data on clinical decisions in nsclc patients with uncommon EGFR mutations. Expert Rev Respir Med (2017) 1:51-5. doi: 10.1080/17476348.2017.1267569

38. Huang SF, Liu HP, Li LH, Ku YC, Fu YN, Tsai HY, et al. High frequency of epidermal growth factor receptor mutations with complex patterns in nonsmall cell lung cancers related to gefitinib responsiveness in taiwan. Clin Cancer Res (2004) 10:8195-203. doi: 10.1158/1078-0432.CCR-04-1245

39. Watanabe S, Minegishi Y, Yoshizawa H, Maemondo M, Inoue A, Sugawara S, et al. Effectiveness of gefitinib against non-small-cell lung cancer with the uncommon EGFR mutations g719x and 1861q. J Thoracic Oncol (2014) 9:18994. doi: $10.1097 /$ JTO.0000000000000048

40. Sharma A, Tan TH, Cheetham G, Scott HS, Brown MP. Rare and novel epidermal growth factor receptor mutations in non-small-cell lung cancer and lack of clinical response to gefitinib in two cases. J Thoracic Oncol (2012) 7:941-2. doi: 10.1097/JTO.0b013e31825134ac

41. Wang X, Li J, Duan Y, Wu H, Xu Q, Zhang Y. Whole genome sequencing analysis of lung adenocarcinoma in xuanwei, china. Thoracic Cancer (2017) 8:88-96. doi: 10.1111/1759-7714.12411

42. Marchetti A, Del Grammastro M, Filice G, Felicioni L, Rossi G, Graziano P, et al. Complex mutations \& subpopulations of deletions at exon 19 of EGFR in nsclc revealed by next generation sequencing: Potential clinical implications. PloS One (2012) 7:e42164. doi: 10.1371/journal.pone.0042164

43. Tannheimer SL, Barton SL, Ethier SP, Burchiel SW. Carcinogenic polycyclic aromatic hydrocarbons increase intracellular ca2+ and cell proliferation in primary human mammary epithelial cells. Carcinogenesis (1997) 18:1177-82. doi: $10.1093 / \mathrm{carcin} / 18.6 .1177$

44. Liu L, Liu X, Ma X, Ning B, Wan X. Analysis of the associations of indoor air pollution and tobacco use with morbidity of lung cancer in Xuanwei, China. Sci Total Environ (2020) 15717:135232. doi: 10.1016/j.scitotenv.2019.135232

45. Kim C, Chapman RS, Hu W, He X, Hosgood HD, Liu LZ, et al. Smoky coal, tobacco smoking, and lung cancer risk in Xuanwei, China. Lung Cancer (2014) 84(1):31-5. doi: 10.1016/j.lungcan.2014.01.004

46. Liu XY, Liu LQ, Zou XN, Ma XY, Ning BF, Ning YF, et al. Epidemiological Features of Lung Cancer Mortality between 1990 and 2016 in Xuanwei City, Yunnan Province. Zhongguo Yi Xue Ke Xue Yuan Xue Bao (2019) 41(3):33843. doi: 10.3881/j.issn.1000-503X.10921

47. Fan X, Wang X, Zhang M, Deng H, Liu Y. Comparison detection methods for EGFR in formalin-fixed paraffin-embedded tissues of patients with NSCLC. Pathol Res Pract (2020) 216(1):152783. doi: 10.1016/j.prp.2019.152783

48. Guibert N, Hu Y, Feeney N, Kuang Y, Plagnol V, Jones G, et al. Ampliconbased next-generation sequencing of plasma cell-free DNA for detection of driver and resistance mutations in advanced non-small cell lung cancer. Ann Oncol (2018) 29(4):1049-55. doi: 10.1093/annonc/mdy005

Conflict of Interest: The authors declare that the research was conducted in the absence of any commercial or financial relationships that could be construed as a potential conflict of interest.

Copyright $\odot 2020 \mathrm{Lv}$, Liu, Liu, Zhang, Jiang, Li, Lu, Lei, Liang and Lin. This is an open-access article distributed under the terms of the Creative Commons Attribution License (CC BY). The use, distribution or reproduction in other forums is permitted, provided the original author(s) and the copyright owner(s) are credited and that the original publication in this journal is cited, in accordance with accepted academic practice. No use, distribution or reproduction is permitted which does not comply with these terms. 